\title{
Newborn BCG Vaccinations: Scar Formation and Tuberculin Conversion Rates: A Retrospective Study
}

\author{
Hanieh Tabatabaei ${ }^{1}$, Sam Hassan ${ }^{2}$ \\ ${ }^{1}$ College of Medicine, Mohammad Bin Rashid University of Medicine and Health Sciences, Dubai, United Arab Emirates \\ ${ }^{2}$ Department of Pediatrics, Mediclinic City Hospital, Dubai, United Arab Emirates
}

Email address:

haniehtabatabaei@mail.com (H. Tabatabaei),samhassan@gmail.com (S. Hassan)

\section{To cite this article:}

Hanieh Tabatabaei, Sam Hassan. Newborn BCG Vaccinations: Scar Formation and Tuberculin Conversion Rates: A Retrospective Study. American Journal of Pediatrics. Vol. 5, No. 1, 2019, pp. 1-6. doi: 10.11648/j.ajp.20190501.11

Received: December 20, 2018; Accepted: January 14, 2019; Published: January 31, 2019

\begin{abstract}
Through this study, we aimed to determine the Bacillus Calmette-Guérin (BCG) vaccination uptake by scar formation rate following newborn BCG vaccinations and tuberculin conversion in a tertiary hospital setting in Dubai, United Arab Emirates. A retrospective cohort analysis was conducted on 945 babies vaccinated with BCG during 1 January and 31 December 2017. All babies were administered with intradermal $0.05 \mathrm{ml}$ of BCG in the left deltoid. The descriptive variables include vaccination coverage. The outcome data includes follow up at 2, 4, and 6 months for scar formation, Mantoux test results, and revaccination data. Out of 945 babies, 911 (96.4\%) were vaccinated by trained nurses with BCG after birth and before being discharged. This was following written consent from parents who were well informed by the details of the vaccinations. The non-vaccinated babies $34(3.6 \%)$ were from different resident nationalities and the parents declined the BCG vaccine. Reason for such decline is not documented but it is likely because in their home country such vaccines are not routinely given. The number of babies who did not return for follow up was $483(53 \%)$. These babies were followed by other centers or outside the country hence were not included in the study. The number of babies followed up with no record on BCG scar was 334 out of the total number of 482 (78\%). BCG scar formation was recorded in 84 babies (19.6\%) followed up. Scar formation failure was recorded in $10(2.3 \%)$. Six babies $(60 \%)$ of those who had no scar had Mantoux test performed. The association between absence of scar and negative Mantoux results were highly significant $(\mathrm{P}<0.05)$ with $100 \%$ of babies having negative Mantoux results, therefore, no immunity. It is concluded that BCG scar is poorly recorded similarly to other high-income countries. There is a good correlation between lack of BCG scar and no immunity to Tuberculosis. Improvement of newborn BCG uptake is importantly needed. It is recommended to introduce mandatory recording of the outcome of BCG scar formation and resultant outcomes in the vaccinations books. Training of personnel administering the BCG vaccination is an important element for successful vaccination in this vulnerable age group.
\end{abstract}

Keywords: BCG, Newborn, Vaccine, Tuberculosis, Mantoux Test, Scar

\section{Introduction}

Tuberculosis (TB) is a serious global health emergency due to its high mortality and morbidity rates [1]. According to the World Health Organization (WHO), TB is one of the top ten causes of deaths worldwide [2-3]. United Arab Emirates (UAE) government considers TB as a major health problem. Dubai Health Authority (DHA) follows a strict guideline for the TB vaccine [4]. TB prevalence remains low in the UAE, estimated $1 / 100,000$, due to mandatory residence screening and vaccination programs. Chest X-rays are done on all expat residents before commencing work in the UAE
[5]. However, confirmed cases of TB are still reported [4-5]. Moreover, the Middle East still remains a region with high TB concern [4, $6-8]$. Immunity against $\mathrm{TB}$ is limited to the Bacillus Calmette-Guérin (BCG) vaccine [9]. The BCG vaccine is a live attenuated vaccine strain developed from Mycobacterium bovis which is commonly found in cows [10]. It produces lifelong cellular immunity against TB and its complications in children including Tuberculous Meningitis which causes serious brain damage [1]. BCG vaccines have also shown to have non-TB benefits in childhood survival rates [11]. A single dose of $0.05 \mathrm{ml}$ in a 26 $\mathrm{G}$ needle is administered in the critical intradermal layer of 
the lateral aspect of the left upper arm at the level of the insertion of the deltoid muscle [10-12]. In the UAE, BCG vaccines are mandatory for babies at birth [5]. The size of the scar formed after the vaccination is a commonly used indicator of immunity. However, the sensitivity of the BCG scar as an index of vaccination status is still the subject of debate. Failure to form a scar may be due to a number of factors including lack of maturation of the immune system, a faulty technique of administration and non-potent vaccines [13]. The Mantoux test (MT) is done to confirm the immunity against TB [1].

The purpose of this study is to determine BCG scar rates and the cases of tuberculin conversion (Mantoux test result changes from negative TB to positive TB within a 24-month period) in the UAE by taking Mediclinic City Hospital $(\mathrm{MCH})$ as an example that is statistically applicable.

Therefore, the objectives of this study are to determine, from the number of babies born, the number of babies administered with BCG vaccinations during the first week of birth. Then to determine the number of BCG scar formation to follow up from the cases of BCG vaccinated babies and analyze Mantoux test results carried out in all babies administered with BCG vaccines who failed to show BCG scar at 2 months, 4 months, or 6 months. All that is to answer one question: what is the outcome of $\mathrm{BCG}$ vaccinations in newborn babies?

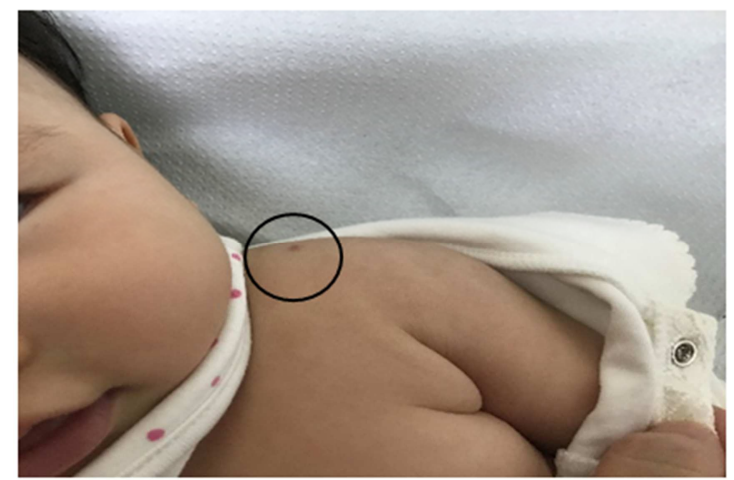

Figure 1. Baby with BCG scar on top of the shoulder due to a faulty technique of administration despite the scar formation. The correct location of the BCG scar should be in the insertion of the deltoid of the left upper arm according to the World Health Organization [2-3].

\section{Material and Methods}

Retrospective cohort data analysis was performed on babies born in MCH from 1 January and 31 December 2017. Data were obtained from a large 280-bed plus tertiary-care private hospital with over 70 specialty departments located in the emirate of Dubai, United Arab Emirates. The hospital follows the DHA guideline for vaccinations. The trained nurses administer the BCG vaccine. The team of pediatricians and specialized nurses fill and approve the vaccination forms. The period of data collection was from 10 June 2018 and 6 September 2018. The data is from 1 January and 31 December 2017.
945 babies out of 4000 born in MCH between 1 January and 31 December 2017 were included in the analysis. The files with babies that were administered with BCG vaccinations during the first week of birth were included. Babies not born in $\mathrm{MCH}$ and children vaccinated with BCG at all other ages were excluded. From these files, the data for scar formation at 2 months, 4 months and 6 months at follow up was selected. From the selected files, the scar formation or lack of was analyzed. In the absence of scar formation, the number of Mantoux test positive (An induration of more than $5 \mathrm{~mm}$ is considered positive) for TB and Mantoux test negative were determined [1]. Variables including decline and revaccination of the BCG were included [Figure 1]. Electronic medical records were obtained from the Hospital Information System (HIS) using the Medical Record Number (MRN) of the babies born in $\mathrm{MCH}$. The vaccination status of the patient and follow up appointments are recorded on the HIS by the attending pediatric physician. The nurse fills out the vaccination form with the time and date of the BCG vaccine. The electronic copy of the vaccination form is kept on the HIS. Taking Confidence level 95, margin error as 5, and power of study as 80 , the sample size of minimum 351 babies was determined [14]. However, since the study is retrospective cohort and follow up times recorded are important and given the time to collect data, 945 files were studied through the process of random sampling. Data were extracted in a Microsoft Excel sheet and transferred to IBM SPSS Statistics 24 for statistical analysis. Qualitative variables were tabulated as frequencies and percentages. Outcome variables were cross-tabulated with factors and characteristics using Chisquare. The alpha value of $p \leq 0.05$ was chosen to determine statistical significance [1, 15 - 16].

\section{Results}

A total of 911 newborn babies from different nationalities were included in the study. The majority were vaccinated with BCG $(96.4 \%)$ after birth while only $3.6 \%(n=34)$ were not vaccinated with BCG by the request of the parents who declined BCG vaccine. The nationalities of babies who were not vaccinated are $51.5 \%$ $(n=17)$ French and $12.1 \% \quad(n=4)$ Canadian. The other babies were American, Australian, and Italian each 2 $(6.1 \%)$ respectively. The remaining were British, Czech, Filipino, Irish, New Zealand, and Syrian each 1 (3.0\%) respectively. One baby not vaccinated with BCG was not documented for nationality and was excluded [Figure 2]. The follow up after birth for vaccinations, growth, and development is not necessarily done in the same facility and this depends on many factors including the insurance, the location of residence, and other factors such as migration. The number of babies followed after birth declined progressively in the first year. More than half $56.1 \%(n=511)$ of babies that were vaccinated were not followed up for scar at 2 months, $62.7 \%(n=572)$ were not 
followed up at 4 months, and $64.4 \%(n=587)$ babies were not followed up for scar at 6 months [Figure 3]. Out of 428 who returned to $\mathrm{MCH}$ for follow up $78.0 \%(\mathrm{n}=334)$ babies had no BCG scar information recorded and $17.7 \%$ $(\mathrm{n}=76)$ babies had a BCG scar at 2 months follow up. While $2.3 \%(n=10)$ babies did not form a BCG scar at 2 months follow up. Two $(0.5 \%)$ babies were noted to have a BCG scar at 4 months. Six (1.5\%) babies were noted to have a BCG scar at multiple months. No babies were recorded for BCG scar at 6 months follow up [Figure 4]. The number of BCG failure rate, confirmed by the lack of scar formation, was $12.0 \%(n=10)$ at 2 months follow up. While the success rate was in $88.0 \%(n=76)$ of the cases. The success and failure rate in 4 months and 6 months is not known since there were no cases of absence of BCG scar formation recorded in those follow-ups. Out of babies with absent BCG scar at 2 months follow up $(n=10)$, $60.0 \%$ of them $(n=6)$ had Mantoux test performed. All 6 $(100.0 \%)$ babies had negative results indicating lack of immunity to BCG. Seven $(70.0 \%)$ babies were revaccinated with BCG vaccine. Two $(28.0 \%)$ babies that were revaccinated were followed up and had a BCG scar.

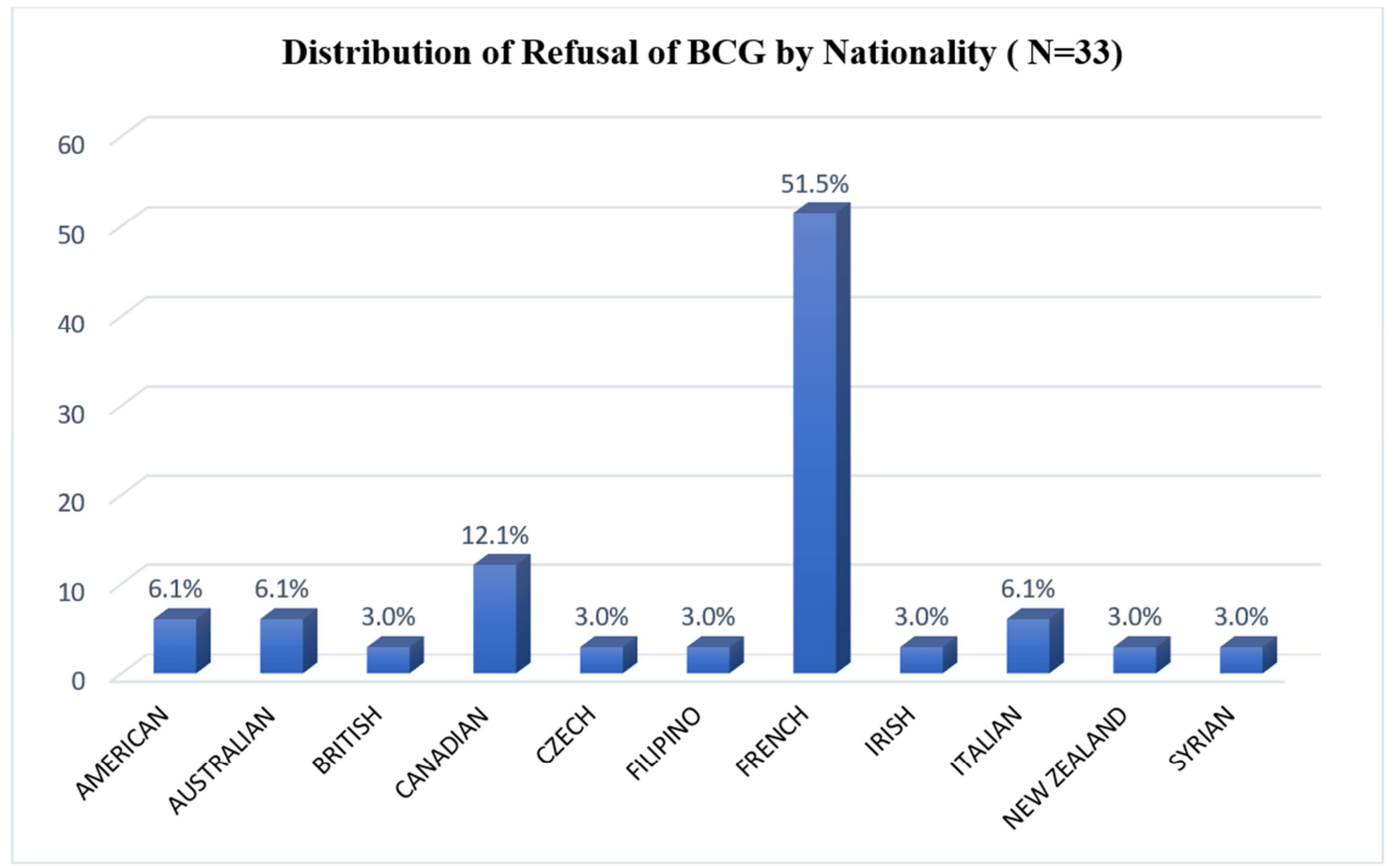

Figure 2. This graph shows the distribution of decline for BCG vaccine by nationality in percentage. The total number of refusals are 33 .

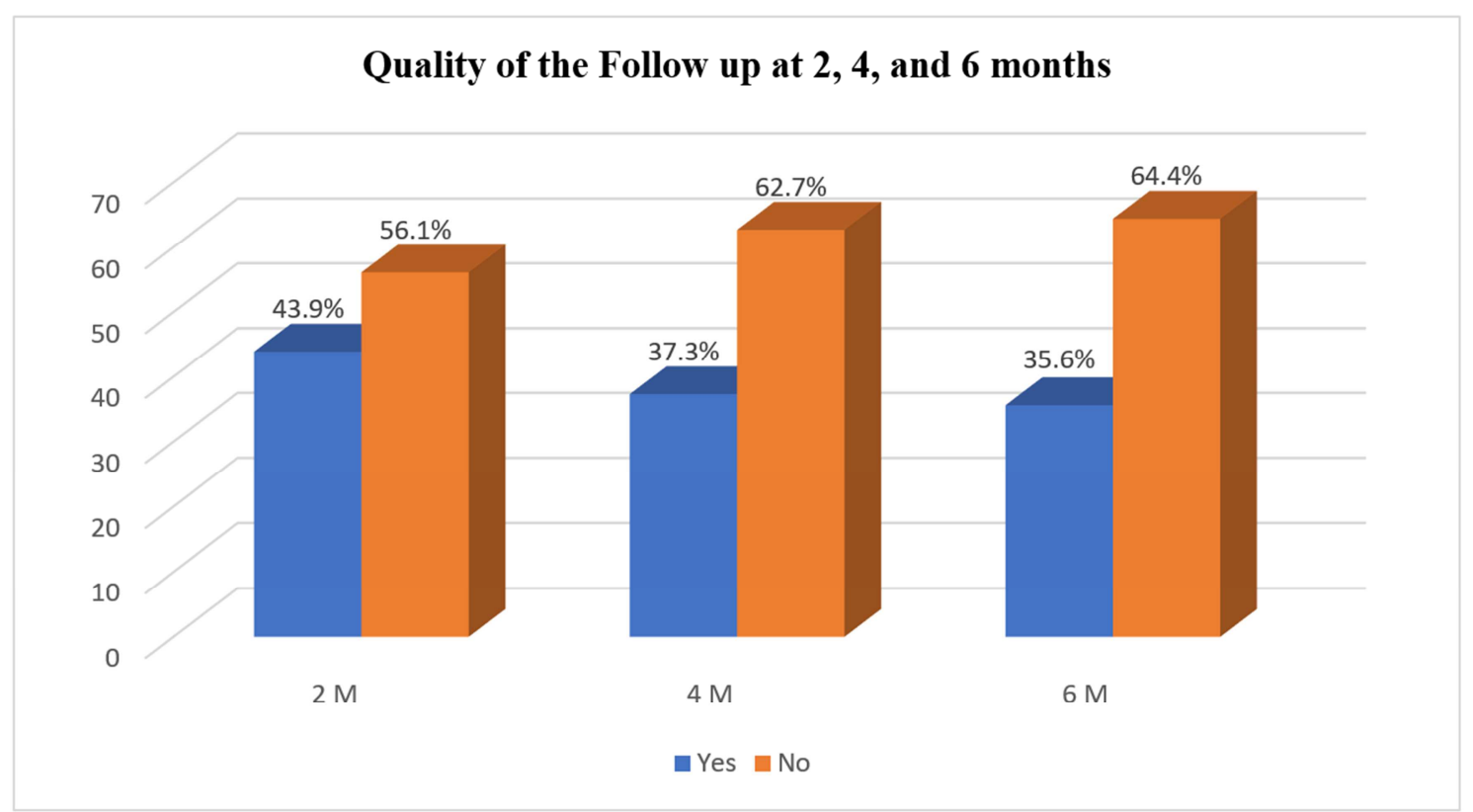

Figure 3. This graph shows the percentage of babies who were followed up at 2, 4 and 6 months. 


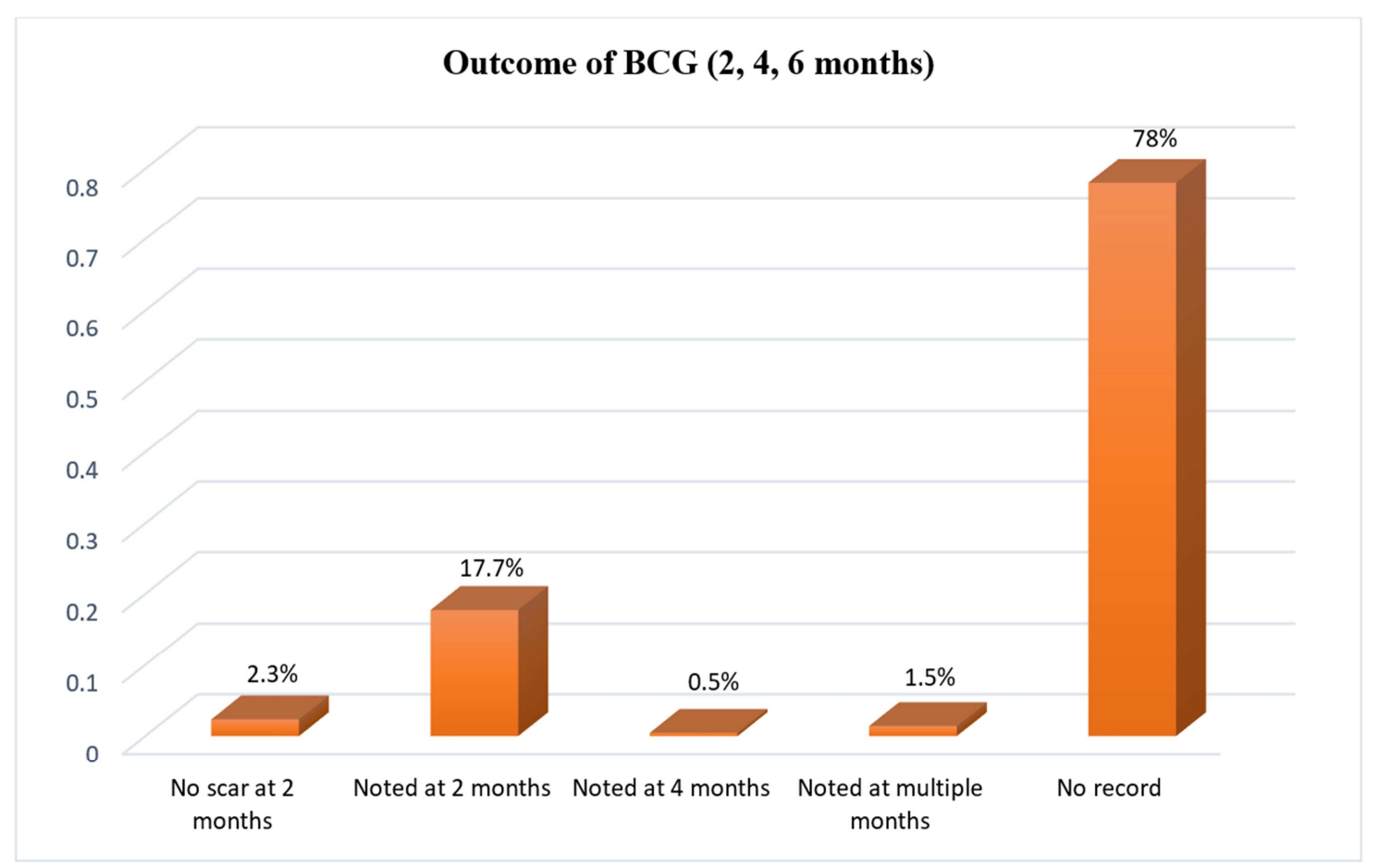

Figure 4. This graph shows the BCG outcome in percentage. The total number of babies followed up were 428.

\section{Discussion}

In the present study, the incidence overall of BCG scar formation in 2017 was 84 babies out of the 428 followed up. The rate of patients not returning for follow up at $\mathrm{MCH}$ were higher than patients returning to $\mathrm{MCH}$ to be followed up for routine 2 months' vaccinations for many reasons that were mentioned before. Most of the babies vaccinated with BCG, who were followed up at 2 months, did not have any record on scar status. In outcomes, $12.0 \%$ of patients that were vaccinated with BCG had not formed a BCG scar at 2 months follow up. $100.0 \%$ of these babies had no immunity to TB due to negative Mantoux test results. This proves the study hypothesis set.

The burden of TB remains high in developing countries and $\mathrm{BCG}$ vaccine is an important armamentarium in preventing $\mathrm{TB}$ in childhood. There are controversies surrounding BCG, however, it is one of the oldest and most commonly used vaccines around the world. BCG is also shown to be protective against tuberculosis meningitis, and miliary TB in children [17-18]. A recent systematic review and meta-analysis of industrialized countries suggest that the incidence of non-tuberculosis mycobacteria lymphadenitis is greatly reduced among BCG-vaccinated children compared with BCG-unvaccinated children [19]. WHO recommends BCG vaccine immediately after birth and before one month after birth. A drawback of this study is the lack of data concerning the time of vaccination for these babies. Due care needs to be taken to vaccinate infants at the earliest. [17]. Our BCG vaccination coverage was $96.4 \%$. This is similar to an extensive study in China in which the BCG coverage was $92.0 \%$ [20]. The rate was lower in Tanzania in which about
$77.0 \%$ were vaccinated with BCG [21]. In the current study, the scar failure rate at 2 months was $12.0 \%$. This is comparable to studies from India with $8.0-10.0 \%$ failure rates. The failure rates are higher in Pakistan with $19.6 \%$ reported. Studies from Brazil had much lower failure rates of $3.1 \%$. LIMA Peru had an even lower failure rate of $1.4 \%$ [1]. The differences in scar failures can be due to different study design and demographic data. The BCG scar success rate depends on many factors including the technique of administration, strain of vaccine, and dose. BCG transportation, storage, and immune disorders in infants also plays a role in scar failure [22]. In this study, all babies were vaccinated with BCG dose $0.05 \mathrm{ml}$ which is the recommended dose $[10,12]$. All children were vaccinated with Indian strain of BCG. There needs to be more research conducted on comparing Indian strain to other strains of BCG. Danish BCG strain is proven to develop a scar more than the Russian BCG strain. The scar size is also larger in the Danish BCG strain [23].

The MT rates were $100.0 \%$ negative in babies with no scar formation. This rate can be biased since only 6 babies did the MT. Regardless, there is an obvious association between the lack of scar and no immunity to TB. This is similar to studies in Taiwan [24]. BCG scar has shown to have decreased mortality in children, this has opened a discussion on revaccinating children who failed to develop a scar after the first BCG vaccination [19]. In our study, all babies with negative MT results were revaccinated.

MT is not as sensitive as Leukocyte Migration Inhibition test (LMIT) in assessing response to BCG. However, it is more accessible and much cheaper. MT and LMIT have also shown good correlation [1]. Only MT was used in our study since it is available and easy to use. 
Our overall scar frequency is $89.0 \%$ from the recorded BCG scar information, which corresponds to scar frequencies found in studies from urban Sub-Saharan Africa (91.0-94.0\%) [25].

These findings suggest that it may be important to monitor the development of a BCG scar as a marker of a wellfunctioning vaccination program [25]. We found that there was an evident number of poor scar records, only $22.4 \%$ of the scar status was recorded amongst the followed up patients. This problem is also seen in researches done in the UK and South India in which a scar reading was poorly concordant with vaccine records [26-27].

There are a number of clinical and strategic implications from the current study findings. Although this study was in a small population, it highlights the need for national implementation by policymakers on the importance of follow up and BCG scar records. This requires all government and private hospitals in the UAE to follow up babies for BCG scar, carry out MT, and revaccinate unimmunized babies. This would increase scar rates and TB immunity in the UAE. There needs to be an emphasis on nurse training. A study in Guinea-Bissau found that $15.0 \%$ of children vaccinated by trained nurses did not develop a scar, while $48.0 \%$ of children vaccinated with BCG at government hospitals were scar-negative. The trained nurses administering the BCG had more BCG scars. This is correlated with better immunity against TB and non-TB mortality in children [28].

The clinicians need to educate parents about the importance of scar formation and TB immunity.

This study reduces this knowledge gap by presenting reference material from a retrospective study on infants assessed within a standardized setup in a high-income country with a low burden of TB. However, it has several limitations: (1) The data were extracted from a lone center from a well-equipped private hospital in Dubai, thus limiting the generalisability of the data to public hospitals in the UAE; (2) The information on factors such as gender, ethnicity, nationality, education status of patients, comorbidities, preterm and term babies, multiple gestations, and vaccination delays were not collected; (3) The small sample size is another key limitation in this study; (4) Parents education on BCG scar formations, as an indication of immunity, cannot be studied in this research. Clearly, this would have an influence in the follow-up rates of the vaccine; (5) The reason behind the decline of treatment with BCG is also unknown. Since the forms have no box to record the cause of the refusal for BCG vaccinations.

This research is amongst the few done on BCG vaccine outcome in the UAE. However, as mentioned in the limitations, the data is extracted from a single private hospital in the emirate of Dubai over a 1-year period (2017). Therefore, the results are not generalizable to all tertiary-care hospitals, private or public, in the UAE and or in the region. Hence as a recommendation, future studies can extract data from multiple private and public hospitals in the UAE to find the BCG outcome, scar failures, and Mantoux test results. In addition, the correlation between training nurses regularly for
BCG administration and BCG scar success rates should be explored. Future clinical trials need to be done on revaccinating infants with scar failure and if it improves mortality in low-income countries.

\section{Conclusion}

This study showed that the majority of BCG vaccinations was not followed up for scarification. Lack of scar formation was associated with no immunity to TB by MT results. There need to be larger studies to reveal the true magnitude of the problem with lack of BCG scar records. We recommended for there to be regular evaluation of BCG vaccination programs. We also recommend adding a distinct section in vaccination booklets for scar status record and MT results.

\section{Acknowledgements}

We gratefully acknowledge staffs in IT and Paediatric departments in Mediclinic City Hospital including the research projects manager Ms. Reem Younis and the HIS training instructor Mr. Deependra Negi. We would also like to acknowledge the research team in MBRU Dr. Aida Azar, Dr. Tom Loney, and Dr. Amar Khamis for their continuous support and guidance.

\section{Conflict of Interest}

The authors declare that there is no conflict of interest regarding the publication of this paper.

\section{Ethical Consideration}

The data was securely saved and studied in the premises of Mediclinic City Hospital (MCH) and all data were anonymous and untraceable back to patients using identifiers. This study was approved by Mohammed Bin Rashid University Institutional Review Board (MBRU-IRBSRP2018-033).

\section{References}

[1] Dhanawade S, Kumbhar S, Gore A, Patil V. Scar formation and tuberculin conversion following BCG vaccination in infants: A prospective cohort study. J Family Med and Prim Care. 2015; 4(3): 384.

[2] Tuberculosis (TB) [Internet]. World Health Organization. 2018 [cited 1 March 2018]. Available from: http://www.who.int/mediacentre/factsheets/fs104/en/.

[3] Trends in Tuberculosis - United States [Internet]. Cdc. 2018 [cited 7 March 2018]. Available from: https://www.cdc.gov/tb/publications/factsheets/statistics/tbtren ds.htm

[4] Immunization Guidelines - Dubai Health Authority [Internet]. [cited 2018Nov24]. Available from:https://www.dha.gov.ae/Documents/Regulations/Guideli nes/Immunization Guidelines.pdf. 
[5] Public Health. Tuberculosis by country: rates per 100,000 people [Internet]. GOV.UK. GOV.UK; 2018 [cited 2018Nov24]. Available from:

https://www.gov.uk/government/publications/tuberculosis-tbby-country-rates-per-100000-people.

[6] Glaziou P, Floyd K, Korenromp EL, Sismanidis C, Bierrenbach AL, Williams BG, et al. Lives saved by tuberculosis control and prospects for achieving the 2015 global target for reducing tuberculosis mortality. Bulletin of the World Health Organization. 2011; 89(8): 573-82.

[7] WHO Global TB Report 2017, progress toward ending TB too slow [Internet]. The Union. [cited 2018Nov24]. Available from: https://www.theunion.org/news-centre/news/the-unionresponse-to-who-global-tuberculosis-report.

[8] Countries with high occurrence of tuberculosis [Internet]. Norwegian Institute of Public Health. [cited 2018Nov24]. Available from: https://www.fhi.no/en/id/infectiousdiseases/TB/countries-tuberculosis/.

[9] Al-Zarouni M, Dash N, Al Ali M, Al-Shehhi F, Pangirahi D. Tuberculosis and MDR-TB in the northern emirates of United Arab Emirates: a 5-year study. The Southeast Asian journal of tropical medicine and public health. 2010; 41(1): 163-168.

[10] BCG Vaccination protocol for newborn infants (CG584) [Internet]. [cited 2018Nov24]. Available from: http://www.royalberkshire.nhs.uk/Downloads/GPs/GP protocols and guidelines/Maternity Guidelines and Policies/Postnatal/BCG for Newborns 2016_V2.0_CG584.pdf.

[11] Roth A, Gustafson P, Nhaga A, Djana Q, Poulsen A, Garly M et al. BCG vaccination scar associated with better childhood survival in Guinea-Bissau. International Journal of Epidemiology. 2005; 34(3): 540-547.

[12] NHS Choices. NHS; [cited 2018Nov24]. Available from: http://www.meht.nhs.uk/about-us-/policies-andguidelines/?assetdet $82735=14766 \& p=22$.

[13] Kheir A, Alhaj A, Ibrahim S. The sensitivity of BCG scar as an indicator of previous vaccination among Sudanese infants. Vaccine. 2011; 29(46): 8189-8191.

[14] Sample Size Calculator [Internet]. Calculator.net. 2018 [cited 1 March 2018]. Available from: http://www.calculator.net/sample-size-calculator.html.

[15] Barton B, Peat J. Medical statistics. Chichester, West Sussex: Wiley Blackwell/BMJ Books; 2014.

[16] Bryman A. Social research methods. Johanneshov: TPB; 2012.

[17] Mcshane H. Tuberculosis Vaccines: Beyond Bacille CalmetteGuerin. Philosophical Transactions of the Royal Society B: Biological Sciences.2011; 366(1579): 2782-2789.
[18] Hassan S, Hassan Abro A. Renal Tuberculosis in a 14-YearsOld Boy Presented as Pyrexia of an Unknown Origin (PUO): A First Case Report. American Journal of Pedriatics. 2018;4(2): 21-24.

[19] Zimmermann P, Finn A, Curtis N. Does BCG Vaccination Protect Against Nontuberculous Mycobacterial Infection? A Systematic Review and Meta-Analysis. The Journal of Infectious Diseases. 2018; 218(5): 679-87.

[20] Hu Y, Chen Y, Liang H, Wang Y. An Overview of Coverage of BCG Vaccination and Its Determinants Based on Data from the Coverage Survey in Zhejiang Province. International Journal of Environmental Research and Public Health. 2018;15(6): 1155 .

[21] Pelzer PT, Mutayoba B, Cobelens FG. BCG vaccination protects against infection with Mycobacterium tuberculosis ascertained by tuberculin skin testing. Journal of Infection. 2018;77(4): 335-40.

[22] Kaur S, Fardi MM, Agarwal KN. BCG vaccination reaction in low birth weight infants. Indian Journal of Medical Research.2002; 116: 64-9.

[23] Frankel H, Byberg S, Bjerregaard-Andersen M, Martins C, Aaby P, Benn C, et al. Different effects of BCG strains - A natural experiment evaluating the impact of the Danish and the Russian BCG strains on morbidity and scar formation in Guinea-Bissau. Vaccine. 2016; 34(38): 4586-93.

[24] Shen CM, Soong WJ, Jeng MJ, Hwang B. Tuberculin response in infants six months after an intradermal Bacille Calmette-Guerin Vaccination. Fu-Jen Journal of Medicine. 2007;5:115-21.

[25] Birk NM, Nissen TN, Ladekarl M, Zingmark V, Kjærgaard J, Jensen TM, et al. The association between Bacillus CalmetteGuérin vaccination (1331 SSI) skin reaction and subsequent scar development in infants. BMC Infectious Diseases. 2017;17(1).

[26] Mangtani P, Nguipdop-Djomo P, Keogh RH, Trinder L, Smith PG, Fine PE, et al. Observational study to estimate the changes in the effectiveness of bacillus Calmette-Guérin (BCG) vaccination with time since vaccination for preventing tuberculosis in the UK. Health Technology Assessment. 2017;21(39):1-54.

[27] Adinarayanan S, Culp RK, Subramani R, Abbas KM, Radhakrishna S, Swaminathan S. Role of bacille CalmetteGuérin in preventing tuberculous infection. International Journal of Tuberculosis and Lung Disease. 2017; 21(4):420424.

[28] Funch KM, Thysen SM, Rodrigues A, Martins CL, Aaby P, Benn CS, et al. Determinants of BCG scarification among children in rural Guinea-Bissau: A prospective cohort study. Human Vaccines \& Immunotherapeutics. 2018;14(10):2 . 